\title{
Transient Abnormal Myelopoiesis with a Novel GATA1 Mutation in a Child with Down Syndrome: A Case Report and Brief Review
}

\author{
Mohanaraj Ramachandran ${ }^{1}$ Prasanth Srinivasan ${ }^{1}$ Jagdish Prasad Meena ${ }^{1}$ \\ Aditya Kumar Gupta ${ }^{1}$ Tanya Prasad ${ }^{2}$ Rachna Seth ${ }^{1}$
}

${ }^{1}$ Department of Pediatrics, Division of Pediatric Oncology, All India Institute of Medical Sciences, New Delhi, India

${ }^{2}$ Laboratory Oncology Unit, Dr. B. R. A. IRCH, All India Institute of Medical Sciences, New Delhi, India

\begin{abstract}
Address for correspondence Jagdish Prasad Meena, MD, Department of Pediatrics, Division of Pediatric Oncology, All India Institute of Medical Sciences, New Delhi 110029, India (e-mail: drjpmeena@yahoo.com).
\end{abstract}

Ind J Med Paediatr Oncol 2021;42:301-304.

\section{Introduction}

Children with Down syndrome (DS) have an increased chance of developing acute leukemia. Acute myeloid leukemia and acute lymphoblastic leukemia risks are increased by 150 and $\sim 30$-fold, respectively, in children with DS. ${ }^{1,2}$ Transient abnormal myelopoiesis (TAM) is characterized by increased circulating blast cells $(>10 \%)$ and GATA1 mutation with or without clinical features and requires close monitoring. ${ }^{3.4} \mathrm{~A}$ few neonates with DS may have small mutant clones of GATA1 along with $<10 \%$ of blasts and these patients have clinically and hematologically silent disease (silent TAM). ${ }^{5}$ In a majority of cases of TAM, complete remission with reversal of GATA1 mutant clone occurs spontaneously without the requirement of chemotherapy. Nevertheless, 10 to $20 \%$ of neonates with TAM consequently develop myeloid leukemia of DS in the first 5 years of life. ${ }^{6}$ The reported mortality rate of TAM is up to $20 \%{ }^{7}$ Here we describe a case of TAM with novel GATA1 mutation who subsequently required chemotherapy and recovered.

\section{Case Report}

\section{Case History}

A 2-month-old male infant, second child born out of a nonconsanguineous marriage to a 22-year-old second gravida mother at term gestation, delivered at home with a birth weight of $2 \mathrm{~kg}$, cried immediately after birth, and had no perinatal complications. The baby was well for the initial 10 days of life, after which he developed cough, coryza, and poor feeding for 5 days with bluish discoloration of lips while crying, inadequate weight gain, forehead sweating, and suck-rest-suck cycle. There was no puffiness of eyes, abdominal distension, progressive pallor, or bleeding manifestations.

The child was admitted to a nearby hospital in the third week of life for these complaints. He underwent echocardiography and was diagnosed to have cyanotic congenital heart disease. He was referred to our hospital for cardiac surgery and further management in the sixth week of life. In preoperative workup at the cardiology unit, the child was found to have a high total leukocyte count (TLC) of 1,29,000

(C) 2021. Indian Society of Medical and Paediatric Oncology. This is an open access article published by Thieme under the terms of the Creative Commons Attribution-NonDerivative-NonCommercial-License, permitting copying and reproduction so long as the original work is given appropriate credit. Contents may not be used for commercial purposes, or adapted, remixed, transformed or built upon. (https://creativecommons.org/licenses/by-nc-nd/4.0/). Thieme Medical and Scientific Publishers Private Ltd. A-12, Second Floor, Sector -2, NOIDA -201301, India 
$\times \mathrm{mm}^{3}$ and transferred to us for further workup in the eighth week of life.

\section{Clinical Findings}

On examination, the child's heart rate was $165 / \mathrm{min}$, respiratory rate was $53 / \mathrm{min}$, and blood pressure was normal with no difference in the four limbs with oxygen saturation of 60 to $63 \%$ in all extremities. The infant's weight was $2.2 \mathrm{~kg}$, length was $47 \mathrm{~cm}$, and head circumference was $33 \mathrm{~cm}$, suggestive of failure to thrive. He had central cyanosis, wide-open anterior fontanelle $(3 \mathrm{~cm} \times 3 \mathrm{~cm})$, with open posterior fontanelle. The baby had a Down phenotype with flat facies, epicanthal eye fold, low set ears, mongoloid slant, clinodactyly, generalized hypotonia, and sandal gap. On cardiovascular examination, the apical impulse was felt in the left 4th intercostal space in the midclavicular line, with a short systolic murmur well heard in the left lower sternal border. The liver was palpable $6 \mathrm{~cm}$ below the right costal margin and the spleen palpable $3 \mathrm{~cm}$ along the splenic axis. The child had tachypnea with mild subcostal and intercostal retractions with normal auscultatory findings, and central nervous examination was within normal limits.

\section{Diagnostic Assessment}

Initial complete blood counts revealed hemoglobin of $14 \mathrm{~g} / \mathrm{dL}$, TLC of $129,000 / \mathrm{mm}^{3}$, platelet count of $2,20,000 / \mathrm{mm}^{3}$, blood urea of $52 \mathrm{mg} / \mathrm{dL}$, serum creatinine of $0.6 \mathrm{mg} / \mathrm{dL}$, uric acid of $6.6 \mathrm{mg} / \mathrm{dL}$, sodium of $135 \mathrm{mEq} / \mathrm{L}$, potassium of $4.7 \mathrm{mEq} / \mathrm{L}$, total protein of $5.2 \mathrm{~g} / \mathrm{dL}$, albumin of $3.8 \mathrm{~g} / \mathrm{dL}$, total bilirubin of $0.9 \mathrm{mg} / \mathrm{dL}$, aspartate transaminase of $31 \mathrm{IU} / \mathrm{L}$, alanine transaminase of $26 \mathrm{IU} / \mathrm{L}$, alkaline phosphatase of $374 \mathrm{IU} / \mathrm{L}$, total calcium of $8.7 \mathrm{mg} / \mathrm{dL}$, and phosphorus of $5.6 \mathrm{mg} / \mathrm{dL}$. His serum thyroid stimulating hormone was high $(8.89 \mathrm{mIU} / \mathrm{L})$; $\mathrm{T} 3(0.57 \mathrm{ng} / \mathrm{mL})$ and T4 level $(4.15 \mu \mathrm{g} / \mathrm{dL})$ were normal. Chest $\mathrm{X}$-ray showed no cardiomegaly, pericardial, or pleural effusion. Echocardiography revealed d-transposition of great arteries with $7 \mathrm{~mm}$ ostium secundum atrial septal defect and $2 \mathrm{~mm}$ patent ductus arteriosus with moderate pulmonary artery hypertension.

His karyotyping was suggestive of trisomy 21. Peripheral smear examination revealed $74 \%$ blasts ( - Fig. $\mathbf{1}$ ), and bone marrow examination revealed 25\% myeloid series blasts. Megakaryocytes appeared reduced, and blasts were negative for myeloperoxidase (MPO) staining. Bone marrow flow
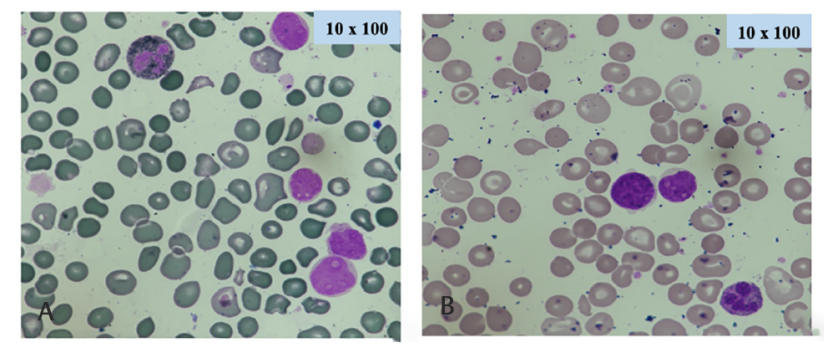

Fig. 1 (A) Giemsa stain of peripheral smear showing blasts having high nuclear-cytoplasmic ratio with cytoplasmic blebs; (B) myeloperoxidase (MPO) stains of peripheral smear showing blasts that are negative for MPO along with neutrophil as control. cytometry revealed $25 \% \mathrm{CD} 45 \mathrm{dim}+$ blasts that were CD34+ (heterogeneous), CD33+ (heterogeneous), CD117+ (heterogeneous), CD38+, HLA-DR+ (heterogeneous), CD56 (heterogeneous), CD7 (heterogeneous), CD36+, CD4+(dim) and negative for cMPO, CCD79a, CD13, CD19, cCD3, sCD3, CD16, CD123, CD11b, CD64, and CD14. A subset of 30\% blasts also express CD34+ (heterogeneous), CD117+ (heterogeneous), cCD41+, cCD61+, CD36 +, CD23a+, and CD71 (dim heterogeneous). Next-generation sequencing (NGS) from the bone marrow aspirate was detected to have novel GATA1 mutation showing a splice variant intron 2 mutation c.220+1 G>T (ENST00000376670.3) (-Fig. 2). This mutation has not been reported in available genomic databases, which may be a novel mutation in TAM.

\section{Therapeutic Intervention}

The child was managed with diuretics and other anticongestive measures with oxygen support for congestive cardiac failure. The child received allopurinol, thyroxine supplementation, and platelet transfusion (for a platelet count of 10,000/ $\mathrm{mm}^{3}$ ). In view of hyperleukocytosis and hepatosplenomegaly leading to respiratory compromise, cytarabine was given at $1 \mathrm{mg} / \mathrm{kg}$ twice a day for 7 days. The child went into remission at 3 weeks, and blasts disappeared. After a stay of 4 weeks, the child was discharged in a stable condition.

\section{Follow-Up and Outcome}

The child is active and doing fine 12 months since the diagnosis of TAM. His latest hemoglobin was $10.9 \mathrm{~g} / \mathrm{dL}$, TLC was $13,450 / \mathrm{mm}^{3}$, and platelet count was $1,50,000 \times 10 \mathrm{~mm}^{3}$.

\section{Discussion}

TAM is a unique entity in DS and is also known as a transient myeloproliferative disorder or transient leukemia of DS. It is a genetic diagnosis characterized by an increased peripheral blood blast count in children with trisomy 21 or mosaic DS with GATA1 mutation with or without clinical features of TAM. ${ }^{7}$ GATA1 mutation and DS are a prerequisite to label as TAM.

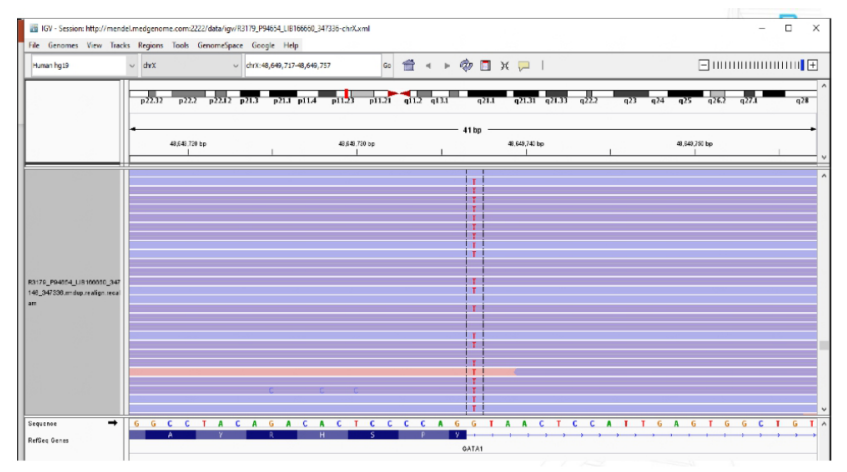

Fig. 2 (Integrative gemomics viewer) snapshot showing intron 2 mutation of the GATA genes. (The nucleotide " $G$ " is altered to " $T$ " allele, leading to a splice variant in the GATA 1 gene. The mutant allele percentage is $77 \%$ that means out of 796 reads, the T allele is present in $77 \%$ of those reads. The annotation of the variant is c. $220+1 \mathrm{G}>\mathrm{T}$.) 
There is no defined blast count cutoff to label as TAM. In the Oxford Imperial DS cohort study with a prospective follow-up of 200 DS neonates, $97.5 \%$ had blasts in the peripheral blood, and $8.5 \%$ had GATA1 mutations. All mutation-positive neonates had a blast count of more than $10 \%$. Three percent of the cohort had blasts more than $10 \%$ but not detected GATA1 mutation. However, none of the mutation-negative children had clinical features of TAM and did not develop myeloid leukemia even after a follow-up of 35 months. ${ }^{5}$ So, a cutoff of $>10 \%$ can be taken to screen for GATA1 mutation in neonates suspected to have TAM. Silent TAM is an entity in which there are no clinical or hematological features of TAM despite minor clones carrying GATA1 mutation. They will have low $(<10 \%)$ peripheral blood blasts. ${ }^{2}$ The risk of developing myeloid leukemia in silent TAM is very rare. ${ }^{7}$

Although the appropriate time for blood smear examination for peripheral blood blasts percentage in DS is 3 days of life, this is often not possible in developing countries. The peripheral blood blasts will clear rapidly, even within a week. Though most of the TAM is usually detected in the first week of life, where $10 \%$ blast percentage is appropriate, the cutoff of $10 \%$ may not be appropriate in infants presenting late. In most cases, TAM resolves by the age of 3 months; some may take up to 6 months. It varies from 2 to 194 days with a mean of 58 days. ${ }^{8,9}$

GATA1 is a member of the GATA family, which has a common zinc finger domain that recognizes the nucleotide sequence motif GATA. ${ }^{10}$ It plays a main role in the hematopoiesis of erythroblasts, megakaryocytes, mast cells, and eosinophils. GATA1 gene is located in the X chromosome. Normal GATA1 protein consists of three domains: an N-terminal zinc finger domain, a C-terminal zinc finger domain, and a transcriptional activation domain $\mathrm{N}$-terminal portion. ${ }^{11}$ The normal GATA1 gene consists of 6 exons. Inherited mutations of GATA1 canlead toX-linked thrombocytopenia,X-linked thrombocytopenia with thalassemia, and some forms of congenital erythropoietic porphyria. ${ }^{12}$ In contrast, the somatic mutations in DS lead to TAM and DS-AMKL. The incidence of somatic mutations is equal in both males and females. ${ }^{12}$ The majority of somatic mutations in DS occur in exon 2 (97\%) and the remaining in exon 3.1, which results in the formation of a truncated GATA1s protein, leading to an aberrant megakaryopoiesis. ${ }^{13}$ GATA1 mutation can be detected by Sanger sequencing, denaturing high-performance liquid chromatography, and NGS. The sensitivity of NGS is superior when compared with other techniques. We are reporting a novel mutation causing a splice variant in Intron 2 c.220+1G $>$ T (ENST00000376670.3) detected by NGS in our index case.

TAM's clinical presentation varies from just an incidental finding to a very sick presentation with multiorgan failure. The usual clinical presentation includes hepatomegaly, splenomegaly, pleural effusion, pericardial effusion, jaundice, ascites, respiratory distress, skin rash, coagulopathy, and multiorgan failure. TAM can manifest in fetal life with hydrops or other manifestations similar to the postnatal presentation. ${ }^{8,14}$ The hematological abnormalities commonly seen are leukocytosis and blasts in peripheral blood. Platelet count may be normal, elevated, or reduced. Thrombocytopenia is not common in neonates with TAM when compared with DS without TAM. None of the clinical features are specific to TAM. Our index case presented with hyperleukocytosis, and he developed thrombocytopenia during the hospital stay.

Flowcytometry of TAM children shows a variable expression of stem cell markers (CD34/117), myeloid markers (CD33/13), platelet glycoproteins (CD36, CD42, CD61), and aberrant expression of CD56 and CD7. It is challenging to distinguish TAM and acute megakaryocytic leukemia (AMKL) from flow cytometry and bone marrow findings. Age of diagnosis can be a clue toward a TAM/AMKL because both are a continuum of the same disease.

More than $80 \%$ of TAM resolve completely, while 20 to $30 \%$ evolve into MDS/DS-AMKL. Remission is observed by normalization of blood counts and blast clearance, followed by the resolution of clinical symptoms like organomegaly. DS-AMKL can occur after remission or progress into AMKL with persistent abnormal blood parameters with an intervening MDS-like picture. Mortality due to TAM constitutes $\sim 20 \%$ primarily due to hepatic fibrosis leading to liver failure. Other causes include cardiorespiratory failure, renal failure, and infection. ${ }^{8}$

Though TAM is a self-limiting disease, delaying treatment when indicated can result in a fatal outcome. Tunstall et al have found that most clinicians are hesitant in starting chemotherapy considering the self-limiting nature of the disease, which leads to a significant delay in the initiation of life-saving treatment. ${ }^{7}$ BFM (Berlin-Frankfurt-Münster) group recommended treatment with low-dose cytarabine 0.5 to $1.5 \mathrm{mg} / \mathrm{kg} /$ day for 3 to 12 days in TAM presenting with thrombocytopenia, cholestasis/liver dysfunction and white blood cell (WBC) count $>50,000 / \mathrm{mm}^{3}$. Low-dose cytarabine was not associated with significant toxicity. ${ }^{7}$

Muramatsu et al have reported that WBC count $>1 \mathrm{lakh} / \mathrm{mm}^{3}$ and anasarca at diagnosis of TAM are the risk factors for early death. They found statistically significant difference in 1 year survival rate in TAM who presented with WBC count of more than $1 \mathrm{lakh} / \mathrm{mm}^{3}$ among those treated with low-dose cytarabine versus untreated (78.3 vs. $38.5 \%) .{ }^{15}$

Children's oncology group recommends treatment in multiorgan failure, WBC count $>100 \times 10^{9} / \mathrm{L}$ or evidence of leukostasis, hepatopathy (characterized by conjugated bilirubin $>83 \mu \mathrm{mol} / \mathrm{L}$, ascites or massive hepatomegaly), massive hepatosplenomegaly causing respiratory or feeding compromise, hydrops fetalis, pleural or pericardial effusions, renal failure, and disseminated intravascular coagulation. Treatment includes low-dose cytarabine in twice daily dose as mentioned above for 5 to 7 days or $3.3 \mathrm{mg} / \mathrm{kg} /$ day as a continuous infusion for 7 days. ${ }^{7}$ Our index case presented with WBC count of more than 1 lakh $/ \mathrm{mm}^{3}$ and hepatosplenomegaly, causing respiratory compromise, and received cytarabine at $1 \mathrm{mg} / \mathrm{kg}$ twice a day for 7 days. In patients with persistent severe liver dysfunction, repeated courses of cytarabine can be given, but treatment should not be given solely based on hepatomegaly because it might take months to resolve. There is no prophylactic role of cytarabine to prevent future occurrence of AMKL. ${ }^{7}$ 
It is advisable to follow up TAM children with three monthly clinical examinations, peripheral smear for the blasts, complete blood count until the age of 2 years, and then six-monthly till 4 years of age. With additional cytogenetic abnormalities, TAM can evolve into myeloid leukemia, usually before the age of 5 years.

\section{Conclusion}

TAM is exclusive to DS and warrants a screening with complete blood counts and peripheral smear examination in the first week of life, preferably by day 3 because of rapid clearance of blasts. Testing for GATA1 mutation is necessary for children with $>10 \%$ blasts for identifying children at a future risk of myeloid leukemia. It is important to know the prevalence and type of GATA1 mutation prevailing in our country as there is a paucity of data regarding GATA1 mutation. Considering the minimal toxicity of low-dose cytarabine and risks outweighing benefits, timely treatment should be initiated when indicated for a better outcome. This case report highlights the novel GATA1 mutation and treatment strategy in TAM.

\section{Authors' Contribution}

MR and PS designed and wrote the manuscript. TP provided the peripheral smear images. JPM, AKG, and RS supervised the manuscript. Finally, all the authors approved the manuscript.

\section{Declaration of Patient Consent}

Legally Authorized Representative (LAR) has given his consent for publication of this paper.

\section{Funding \\ None}

\section{Conflict of Interest}

No conflicts of interest.

\section{References}

1 Hasle $\mathrm{H}$, Clemmensen IH, Mikkelsen M. Risks of leukaemia and solid tumours in individuals with Down's syndrome. Lancet 2000;355(9199):165-169
2 Patja K, Pukkala E, Sund R, Iivanainen M, Kaski M. Cancer incidence of persons with Down syndrome in Finland: a population-based study. Int J Cancer 2006;118(7):1769-1772

3 Malinge S, Izraeli S, Crispino JD. Insights into the manifestations, outcomes, and mechanisms of leukemogenesis in Down syndrome. Blood 2009;113(12):2619-2628

4 Roberts I, Izraeli S. Haematopoietic development and leukaemia in Down syndrome. Br J Haematol 2014;167(5):587-599

5 Roberts I, Alford K, Hall G, et al. Oxford-Imperial Down Syndrome Cohort Study Group. GATA1-mutant clones are frequent and often unsuspected in babies with Down syndrome: identification of a population at risk of leukemia. Blood 2013;122(24):3908-3917

6 Yoshida K, Toki T, Okuno Y, et al. The landscape of somatic mutations in Down syndrome-related myeloid disorders. Nat Genet 2013;45(11):1293-1299

7 Tunstall O, Bhatnagar N, James B, et al. British Society for Haematology. Guidelines for the investigation and management of transient leukaemia of Down syndrome. $\mathrm{Br}$ J Haematol 2018;182(2):200-211

8 Bhatnagar N, Nizery L, Tunstall O, Vyas P, Roberts I. Transient abnormal myelopoiesis and AML in Down syndrome: an update. Curr Hematol Malig Rep 2016;11(5):333-341

9 Homans AC, Verissimo AM, Vlacha V. Transient abnormal myelopoiesis of infancy associated with trisomy 21. Am J Pediatr Hematol Oncol 1993;15(4):392-399

10 Cantor AB. GATA transcription factors in hematologic disease. Int J Hematol 2005;81(5):378-384

11 Miyauchi J. Unique Myeloid Leukemias in Young Children with Down Syndrome: Cell Origin, Association with Hematopoietic Microenvironment and Leukemogenesis. Prenat Diagn Screen Syndr [Internet]. 2011 Aug 17 [cited 2020 Dec 19]; Available from: https://www.intechopen.com/books/prenatal-diagnosis-and-screening-for-down-syndrome/unique-myeloid-leukemias-in-young-children-with-down-syndrome-cell-origin-association-with-hematopoi. Accessed July 5, 2021

12 Ciovacco WA, Raskind WH, Kacena MA. Human phenotypes associated with GATA-1 mutations. Gene 2008;427(1-2):1-6

13 Alford KA, Reinhardt K, Garnett C, et al. International Myeloid Leukemia-Down Syndrome Study Group. Analysis of GATA1 mutations in Down syndrome transient myeloproliferative disorder and myeloid leukemia. Blood 2011;118(8):2222-2238

14 Singh A, Mandal A, Guru V, Srinivasan S, Seth R. Transient abnormal myelopoiesis: a varied spectrum of clinical presentation. J Hematol (Brossard 2017;6(1):25-28

15 Muramatsu H, Watanabe T, Hasegawa D, et al. Prospective study of 168 infants with transient abnormal myelopoiesis with Down syndrome: Japan Pediatric Leukemia/Lymphoma Study Group, TAM-10 Study. Blood 2015;126(23):1311 